SUBJECT AREAS:

ELECTRONIC PROPERTIES

AND MATERIALS

NANOWIRES

SCIENTIFIC REPORTS:

$4: 3609$

DOI: $10.1038 /$ srep03609

(2014)

Published:

9 January 2014

Updated:

22 May 2014

\section{ERRATUM: Substrate-induced Band Gap Renormalization in Semiconducting Carbon Nanotubes}

Nicholas A. Lanzillo, Neerav Kharche \& Saroj K. Nayak

This Article contains an error in the affiliations listed for the authors Neerav Kharche and Saroj K. Nayak. The correct affiliations are listed below.

Neerav Kharche ${ }^{1,2}$ \& Saroj K. Nayak ${ }^{1,3}$

${ }^{1}$ Department of Physics, Applied Physics, and Astronomy, Rensselaer Polytechnic Institute, 110 8th Street, Troy, NY 12180,

${ }^{2}$ Computational Center for Nanotechnology Innovation, Troy, NY 12180, USA,

${ }^{3}$ School of Basic Sciences, Indian Institute of Technology, Bhubaneswar, India 751007. 